\title{
miR-23b-3p suppressing PGC1a promotes proliferation through reprogramming metabolism in osteosarcoma
}

\author{
Ran Zhu', Xinpan $\mathrm{Li}^{2}$ and Yanhong $\mathrm{Ma}^{1}$
}

\begin{abstract}
Metabolic shift from oxidative phosphorylation (OXPHOS) to glycolysis is a hallmark of osteosarcoma (OS). However, the mechanisms of the metabolic switch have not been completely elucidated. Here we reported that the miR-23b-3p was significantly upregulated in OS cells. Functional studies suggested that knockdown of miR-23b-3p could inhibit OS cell proliferation in vitro or in vivo. In addition, suppression of miR-23b-3p could lead to upregulation of OXPHOS and suppression of glycolysis. Mechanistically, miR-23b-3p promoted OS cell proliferation and inhibited OXPHOS in OS, at least in part, by directly targeting peroxisome proliferator-activated receptor gamma coactivator-1 alpha (PGC1a) and inhibiting its expression. Our data highlights important roles of miR-23b-3p and PGC1a in glucose metabolism reprogram of OS. The suppression of miR-23b-3p may provide effective therapeutic strategies for the treatment of OS.
\end{abstract}

\section{Introduction}

Osteosarcoma (OS) is a common malignant bone tumor and is the main cause of tumor-related deaths in children and adolescents ${ }^{1}$. Over the past 30 years, the development of chemotherapeutics and surgical operation have significantly improved the outcomes of OS patients ${ }^{2}$. However, still $30-40 \%$ of patients with OS develop recurrent or metastatic diseases, which results in poor outcomes ${ }^{3}$. Therefore, it is critical to identify the underlying mechanisms for OS development and progression, and explore new prognostic biomarkers as well as therapeutic targets for more effective therapeutic strategies.

MicroRNAs (miRNAs) are a kind of small non-coding RNA molecules (21-23 nucleotides), which regulate gene expression by binding to the $3^{\prime}$-untranslated regions $\left(3^{\prime}\right.$ UTR) of target mRNA and promoting target mRNA degradation or translational inhibition ${ }^{4}$. Previous studies

Correspondence: Yanhong Ma (mayanhong111@126.com)

'Department of Rehabilitation Medicine, Shanghai Jiao Tong University Affiliated Sixth People's Hospital, Shanghai, China

²Department of Rehabilitation Medicine, Putuo People's Hospital Affiliated to Tongji University, Shanghai, China

Edited by A. Finazzi-Agrò identified that miRNAs could act as oncogenes or cancer inhibitors in various tumors and regulated a wide range of important tumor cell processes including cell proliferation, metastasis, apoptosis, and metabolic reprogramming $^{5-7}$. The miR-23b-3p belongs to the miR-23b/27b/ 24-1 cluster and has been reported to function as an onco-miR in different cancers including glioma, gastric cancer, and breast cancer ${ }^{8-10}$. However, the functions and mechanisms of miR-23b-3p in OS have not been previously reported.

Ongoing studies have revealed that suppression of oxidative phosphorylation (OXPHOS) along with enhanced glycolysis, which is also called the Warburg effect, lead to chemoresistance, proliferation, and metastasis of cancer cells ${ }^{11}$. Peroxisome proliferator-activated receptor gamma coactivator-1 alpha (PGC1 $\alpha$ ) is a multi-functional transcriptional coactivator, which ensures maintenance of mitochondrial homeostasis and controls oxidative metabolism $^{12}$. It has been reported that overexpression of PGC1 $\alpha$ could lead to enhancement of mitochondrial OXPHOS and reduction of aerobic glycolysis, which is also termed anti-Warburg effect, in several types of

\section{(c) The Author(s) 2019}

(c) (i) Open Access This article is licensed under a Creative Commons Attribution 4.0 International License, which permits use, sharing, adaptation, distribution and reproduction cc) in any medium or format, as long as you give appropriate credit to the original author(s) and the source, provide a link to the Creative Commons license, and indicate if changes were made. The images or other third party material in this article are included in the article's Creative Commons license, unless indicated otherwise in a credit line to the material. If material is not included in the article's Creative Commons license and your intended use is not permitted by statutory regulation or exceeds the permitted use, you will need to obtain permission directly from the copyright holder. To view a copy of this license, visit http://creativecommons.org/licenses/by/4.0/. 
tumors ${ }^{13,14}$. However, whether PGC1 $\alpha$ is taken part in the glucose metabolism reprogram in OS remains unclear.

Here we demonstrated that miR-23b-3p is upregulated in OS cells. miR-23b-3p suppressed OXPHOS and promoted aerobic glycolysis in OS, resulting in the enhancement of OS cell proliferation. Furthermore, we identified potential target genes of miR-23b-3p and found that miR-23b-3p inhibited OS cell OXPHOS and growth, at least in part, by directly targeting PGC1 $\alpha$ and inhibiting its expression.

\section{Results}

MiR-23b-3p is upregulated in OS cell lines and promotes the proliferation of os cells

First, we tested the expression level of miR-23b-3p in human OS cell lines. Four human OS cell lines, MNNGHOS, U-2OS, MG63, and Saos-2, as well as one line of human osteoblast cell, hFOB1.19, were tested via quantitative real-time PCR. As shown in Fig. 1a, miR-23b-3p expression level in the OS cell lines was significantly higher than that in the osteoblast cell line. To further investigate the potential function of miR-23b-3p in OS cells, we constructed miR-23b-3p stable knockdown OS cell lines (MNNG-HOS and MG63) via short hairpin RNA (shRNA) method (Fig. 1b). First, we tested whether the knockdown of miR-23b-3p affected the proliferation of OS in vitro. Cell counting kit-8 (CCK-8) assays showed that downregulating miR-23b-3p significantly inhibited cell proliferation of MNNG-HOS and MG63 cells (Fig. 1c, d). Furthermore, compared with the control cells, knockdown of miR-23b-3p also reduced the number of cell colonies of OS cells (Fig. 1e). In addition, we evaluated the cell cycle distribution of miR-23b-3p-knockdown MNNG-HOS and MG63 cells, and found that the G1phase population was increased, whereas the S-phase population was decreased compared with their negative control (NC) cells using flow cytometry (Fig. 1f). To explore the effects of miR-23b-3p on proliferation of OS cells in vivo, we established mouse xenograft models by injecting the control MNNG-HOS cells, as well as stable miR-23b-3p-knockdown MNNG-HOS cells into nude mice subcutaneously. The tumors with miR-23b-3p knockdown displayed an obvious delay in growth (Fig. $1 \mathrm{f}-\mathrm{i})$. In addition, immunohistochemical staining also showed a reduced expression of Ki67 in the miR-23b-3pknockdown tumors (Fig. 1j). These findings suggested that miR-23b-3p promoted the proliferation of OS cells.

\section{miR-23b-3p inhibits OXPHOS and facilitates aerobic glycolysis in OS}

There is no doubt that metabolic shift from OXPHOS to glycolysis promotes rapid proliferation of cancer cells ${ }^{13}$. To assess the effect of miR-23b-3p on glycolytic and mitochondrial flux in OS cells, we initially measured oxygen consumption rate (OCR, a marker of OXPHOS) and extracellular acidification rate (ECAR, a marker of glycolysis) of OS cells by using a seahorse 96XF Extracellular Flux Analyser. As shown in Fig. 2a, knockdown of miR-23b-3p enhanced both basal and maximal OCR in OS cells. In contrast, the basal and maximal ECAR were reduced when miR-23b-3p was silenced in OS cells (Fig. $2 b)$. In addition, knockdown of miR-23b-3p in OS cells also elevated the formation of ATP produced by OXPHOS and reduced the level of lactate produced by the glycolysis (Fig. 2c, d). Collectively, these results demonstrated that miR-23b-3p inhibited OXPHOS and promoted aerobic glycolysis in OS cells.

\section{miR-23b-3p directly targeted PGC1a in OS cells}

To explore the underlying mechanisms through which miR-23b-3p exerts its functional effects in OS cells, we predicted potential targets using two commonly used prediction algorithms TargetScan and miRDB. We found ten glycolysis or OXPHOS-related potential targets genes of miR-23b-3p (Fig. 2e). We compared the mRNA level of potential target genes between control group and miR23b-3p-knockdown group in MNNG-HOS and MG63 cells. As revealed in Fig. 2f, g, knockdown of miR-23b-3p significantly suppressed mRNA expression of PGC1 $\alpha$. Furthermore, previous study had also predicted PGC1 $\alpha$ as a potential target of miR- $23^{15}$. The potential target sites of miR-23b-3p on PGC1 $\alpha$ was shown in Fig. $2 h$. To further confirm that miR-23b-3p could directly target PGC1 $\alpha$, dual luciferase reporter assays were performed. As shown in Fig. 2i, j, miR-23b-3p mimic led to a remarkable decrease in luciferase activity of wild-type PGC1 $\alpha$ 3'-UTR (WT) reporter, but had no obvious effect on the luciferase activity in Mut 3'-UTR of PGC1 $\alpha$ reporter in MG63 and MNNG-HOS cells. Taken together, our results indicated that PGC1 $\alpha$ is a direct target of miR-23b-3p in OS cells.

\section{PGC1a promoted anti-Warburg effect and suppressed the proliferation of OS cells}

PGC1 $\alpha$ was an indicator of mitochondrial biogenesis and OXPHOS, and had been reported to inhibit the growth of a number of cancers, such as hepatocellular carcinoma, renal cell carcinoma, malignant fibrous histiocytoma, and lung cancer ${ }^{9,16-18}$. However, the function of PGC1 $\alpha$ in OS remains unclear. To explore the potential function of PGC1 $\alpha$ in OS cells, we constructed PGC1 $\alpha$ stable overexpression of MG63 and MNNG-HOS cell lines (Fig. 3a). The results of CCK-8 assay and colonyformation assay indicated that upregulation of PGC1 $\alpha$ remarkably inhibited the proliferation of OS (Fig. 3b-d). Upregulation of PGC1 $\alpha$ also promoted the OXPHOS and inhibited aerobic glycolysis in OS cells (Fig. 3e, f). Furthermore, overexpression of $\mathrm{PGC} 1 \alpha$ also elevated the formation of ATP produced by OXPHOS and reduced the 


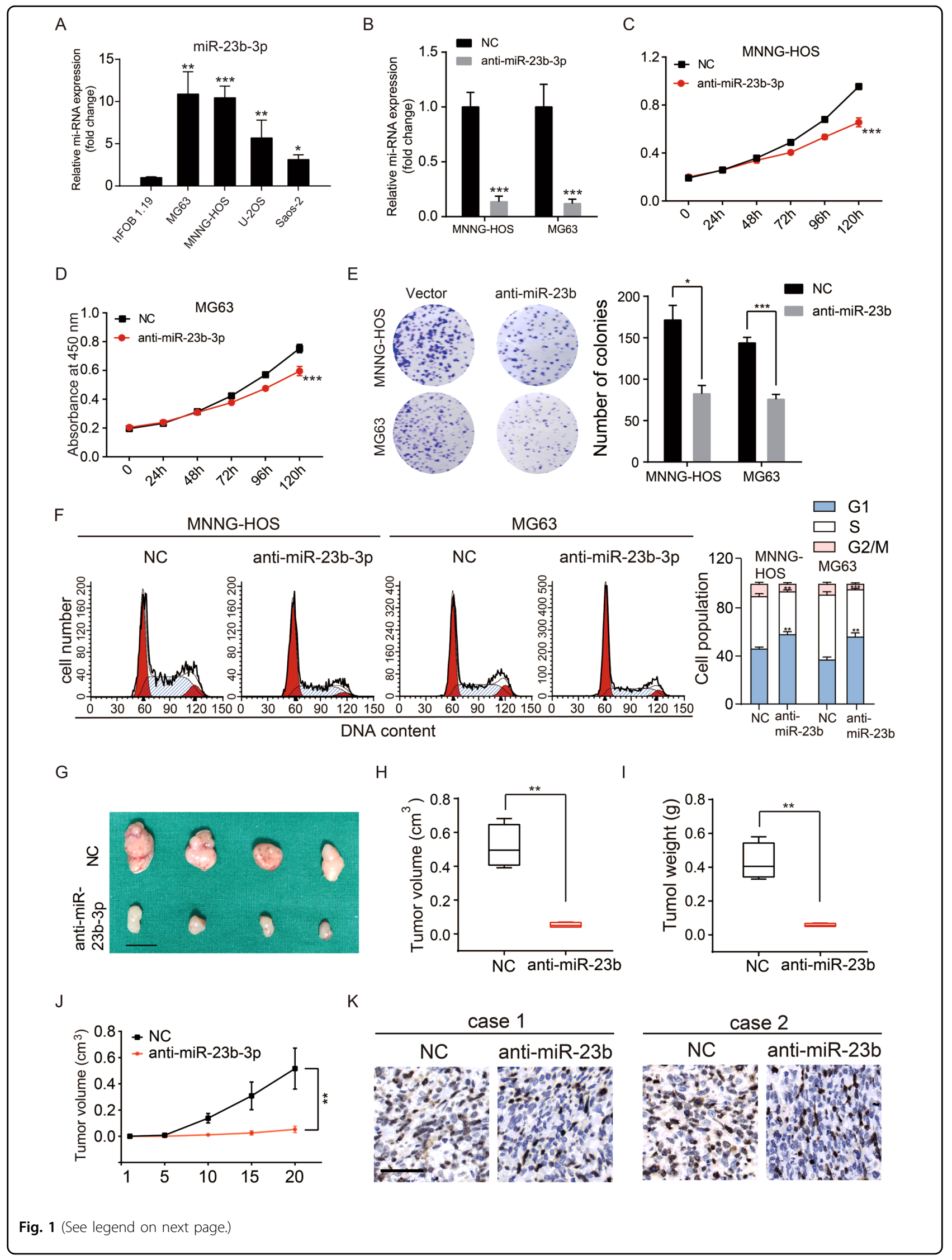


(see figure on previous page)

Fig. 1 miR-23b-3p promoted OS proliferation in vitro and in vivo. a The expression patterns of miR-23b-3p in normal osteoblast cell line (hFOB1.19) and OS cell lines (MNNG-HOS, U-2OS, Saos-2, and MG63) by qRT-PCR. ${ }^{*} p<0.05,{ }^{* *} p<0.01,{ }^{* * *} p<0.001$. b Knockdown efficacy of miR-23b$3 p$ in wild OS cells (MNNG-HOS and ${ }^{*} p<0.05,{ }^{* *} p<0.01,{ }^{* * *} p<0.001$. MG63) was determined by qRT-PCR, ${ }^{* * *} p<0.001$. c, d. Knockdown of miR-23b$3 p$ inhibited MNNG-HOS and MG63 cell proliferation using the cell counting kit (CCK)-8 assay. Values are means \pm SD, ${ }^{* * *} p<0.001$. e Downregulation of miR-23b-3p suppressed OS cell (MNNG-HOS and MG63 cells) proliferation using colony formation assay. Values are means $\pm \mathrm{SD},{ }^{*} p<0.05,{ }^{* * *} p<$ 0.001. f Knockdown of miR-23b-3p led to G1-phase cell cycle arrest and decreased S-phase cell population through using cell cycle analysis. Values are means $\pm S D,{ }^{* *} p<0.05,{ }^{* * *} p<0.001$. g Morphologic characteristics of excised tumors from nude mice in MNNG-HOS/control group and MNNG$\mathrm{HOS} / \mathrm{miR}-23 \mathrm{~b}-3 p$ knockdown group $(n=4)$. Scale bars $=1 \mathrm{~cm}$. $\mathbf{h}$ Tumor weight in miR-23b-3p overexpression group was reduced compared with control group $(n=4),{ }^{* *} p<0.01$. i Tumor volume in miR-23b-3p overexpression group was reduced compared with control group $(n=4)$, ${ }^{* *} p<0.01$. j Tumor volumes were measured with calipers every 5 days. The growth rate in miR-23b-3p knockdown group was significantly slower than that in control group $(n=4),{ }^{* *} p<0.01$. k Representative images of Ki67 staining in tissues from miR-23b-3p knockdown and control mice. Compared with control mice, decreased expression of Ki67 (upper panel) were observed. Scale bars $=50 \mu \mathrm{m}$

level of lactate produced by the glycolysis (Fig. 3g, h). The prognostic significance of PGC1 $\alpha$ in patients with OS was determined by using Kaplan-Meier analysis. Data on PGC1 $\alpha$ were obtained from 88 OS patients from the R2 database. As shown in Fig. 3i, high PGC1 $\alpha$ expression contributed to a better metastasis-free survival rate in OS patients. These data illustrated that PGC1 $\alpha$ inhibited the proliferation of OS through promoting the switch of aerobic glycolysis to OXPHOS.

\section{Knockdown of PGC1a reversed the promotion on OXPHOS and inhibition on aerobic glycolysis and cell proliferation caused by miR-23b-3p knockdown}

We had demonstrated that miR-23b-3p promoted the proliferation and aerobic glycolysis of OS cells (Fig. 1); thus, we investigated whether the phenomenon regulated by miR-23b-3p was relied on its target PGC1 $\alpha$. We first downregulated miR-23b-3p together with PGC1 $\alpha$ in MNNG-HOS and MG63 cell lines (Fig. 4a). As shown in Fig. $4 b-d$, knockdown of PGC1 $\alpha$ diminished the inhibitory effect of miR-23b-3p knockdown on OS cells growth in vitro. In the in vivo assays, a compromised tumorigenic potential in miR-23b-3p knockdown group was partly offset via the downregulation of PGC1 $\alpha$ (Fig. 4e, f). Similarly, the knockdown of PGC1 $\alpha$ partly rescued the aerobic glycolysis in MNNG-HOS and MG63 cells (Fig. $4 \mathrm{~g}, \mathrm{j})$. Taken together, these data suggested that miR-23b$3 p$ promotes aerobic glycolysis and tumor growth by directly targeting PGC1 $\alpha$.

\section{Discussion}

Recently, miR-23b-3p has been specifically linked to various functions in different cancers through a cell-typedependent manner. In several kinds of tumors, miR-23b$3 \mathrm{p}$ was reported to act as a tumor inhibitor. Huang et al. ${ }^{19}$ showed that downregulation of miR-23b-3p in gastric carcinoma inhibited growth and invasion of gastric cancer cells through targeting Notch 2 and inhibiting its expression. miR-23b-3p inhibited tumorigenesis of prostate cancer by repressing Src kinase ${ }^{20}$. Moreover, miR-23b-3p acted as a tumor inhibitor by targeting Zeb1 in bladder cancer ${ }^{21}$.

However, miR-23b-3p was also related to the poor prognosis and acted as a cancer promoter in a number of tumors. $\mathrm{Hu}$ et al. ${ }^{9}$ introduced the hypothesis that miR23b-3p functions as anti-apoptotic factor in gastric cancer cells by directly targeting Programmed cell death protein 4 , an apoptosis regulatory protein. In breast cancer cells, miR-23b-3p negatively regulates Nischarin, an intracellular protein that acts as a tumor suppressor by regulating the metastatic behavior of tumor cells ${ }^{10}$. Moreover, Zaman et al. ${ }^{17}$ showed that upregulation of miR-23b promoted the migration of renal carcinoma cells through targeting Phosphatase and Tensin Homolog (PTEN). Here we demonstrated that miR-23b-3p was upregulated in OS cell lines and promoted the growth of OS cell lines (MNNG-HOS and MG63). Different from our findings, Liu et al. ${ }^{22}$ reported that miR-23b-3p inhibited the proliferation of OS cell line (U-2OS) through targeting SIX1 and inhibiting its expression, as it is well acknowledged that OS have multiple rearrangements across the genome, kataegis, and a high degree of intra- and intertumor heterogeneity $^{23-25}$. The study of Christopher et al. ${ }^{26}$ also highlighted heterogeneity in growth rates and genetics among OS cell lines. Thus, the difference between these results may be due to a high-degree heterogeneity between OS cell lines. More OS cell lines would be used in future studies to further explore the biological functions of miR-23b-3p in OS.

A glucose metabolism characteristic of most cancer cells is the favor of glycolysis for ATP production rather than OXPHOS even when oxygen is abundant, which also was called Warburg effect ${ }^{27}$. There was no doubt that enhanced aerobic glycolysis or Warburg effect promoted rapid proliferation in cancer cells through increasing biosynthesis of macromolecules, enhancing disruption of tissue architecture and immune cell evasion, and so on ${ }^{28}$. Thus, insights into this characteristic alteration and 
A

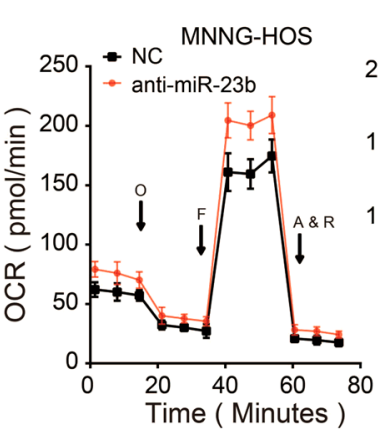

C

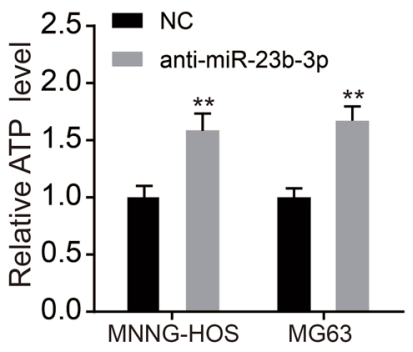

$\mathrm{F}$

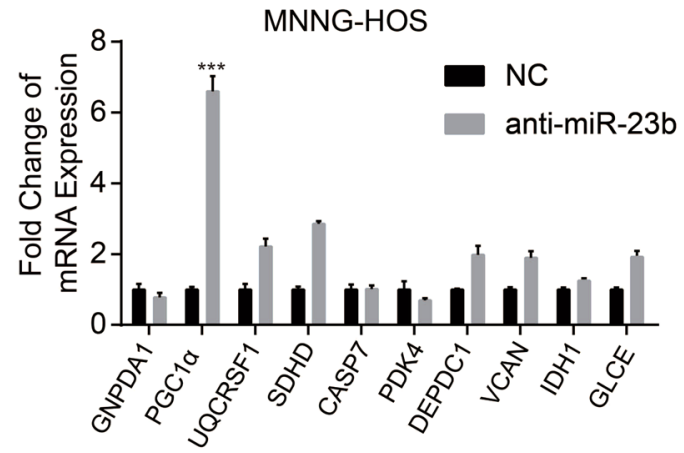

B

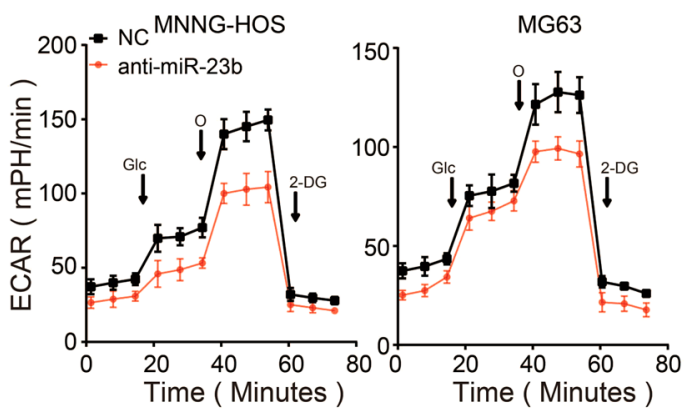

$E$

$\mathrm{D}$

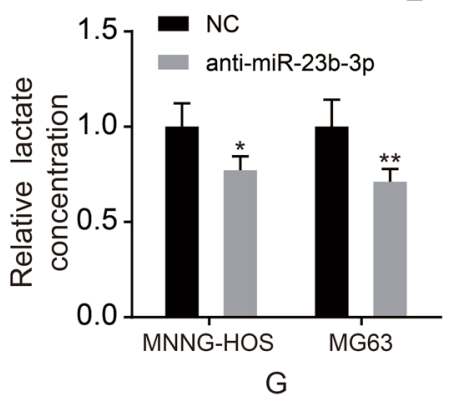

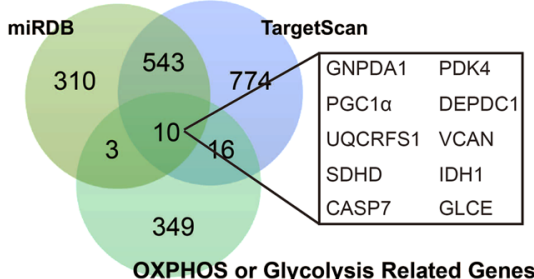

MG63

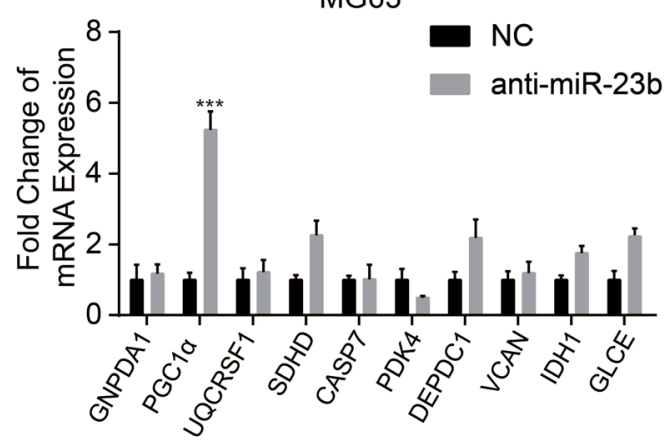

$\mathrm{H}$

Wild-type (WT) PGC1a site 1 3'UTR : 5'AUAGCCAUGUACUAUAATGTGAU 3 '

miR-23b-3p : 3' UTR : 3' CCAUUAGGGACCGUUACACUA 5'

Mutated (MUT1) PGC1a site 13 'UTR : 5 'AUAGCCAUGUACUAUAGCGCAAU 3'

Wild-type (WT) PGC1a site 23 'UTR : 5 ACAGACAUUUUCAAUAATGTGAA 3

miR-23b-3p : 3 UTR : 3 ' CCAUUAGGGACCGUUACACUA 5 '

Mutated (MUT2) PGC1a site 23 'UTR : 5 'ACAGACAUUUUCAAUAGCGCAAA 3'
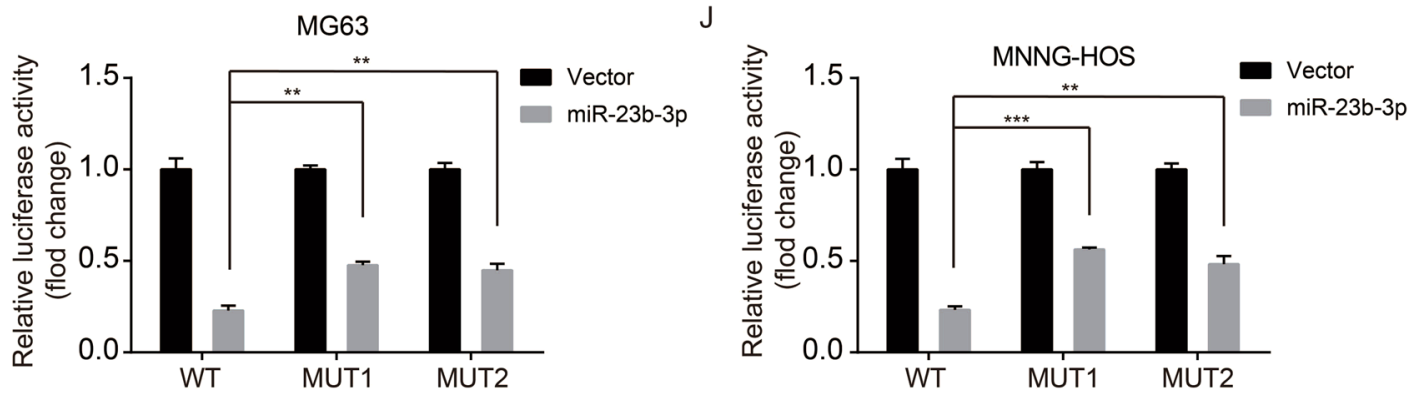

Fig. 2 (See legend on next page.) 
(see figure on previous page)

Fig. 2 miR-23b-3p inhibited OXPHOS and facilitated the aerobic glycolysis in OS cells and directly targeted PGC1a. a $\mathrm{O}_{2}$ consumption rate (OCR) of MNNG-HOS and MG63 cells in control and miR-23b-3p kncokdown group was detected via a Seahorse Bioscience XFp analyzer. Glc: glucose, Oligo: oligomycin, 2-DG: 2-deoxy-d-glucose. $\mathbf{b}$ Extracellular acidification rate (ECAR) of MNNG-HOS and MG63 cells in control and miR-23b-3p knockdown group was detected using a Seahorse Bioscience XFp analyzer. O: Oligomycin, F: FCCP, A\&R: antimycin A/rotenone. c, d. ATP level and lactate production was determined in MNNG-HOS and U-2OS cells stable knockdown miR-23b-3p. Values are means $\pm \mathrm{SD},{ }^{*} p<0.05,{ }^{* *} p<0.01$. e Venn diagram showing the predicted glycolysis or oxidative phosphorylation (OXPHOS)-related target genes of miR-23b-3p from databases (miRDB and TargetScan). $\mathbf{f}, \mathbf{g}$. The mRNA expression patterns of the predicted target genes of miR-23b-3p were determined by qRT-PCR in anti-miR-23b or Control MNNG-HOS and MG63 cells. $\mathbf{h}$ The wild-type and the mutated sequences of the PGC1a mRNA $3^{\prime}$-UTR (mutation site: red). $\mathbf{i}$, j. The luciferase activity of the OS cells (MNNG-HOS and MG63) in luciferase reporter plasmid containing wild-type PGC1a $3^{\prime}$-UTR (Wt) and mutant PGC1a 3'-UTR (Mut1 and Mut2) co-transfected with miR-23b-3p mimics or a negative control was assessed, ${ }^{* *} p<0.01,{ }^{* * *} p<0.001$

promoting a switch from aerobic glycolysis to OXPHOS will hold promise for potential cancer therapy ${ }^{29}$. However, little is known about the role of miRNA in glucose metabolism reprogramming of OS. Here we reported that miR-23b-3p acts as a novel promoter of Warburg effect by targeting PGC1 $\alpha$ directly and inhibiting its expression in OS.

OXPHOS dysfunction is known to lead to compensatory glycolysis ${ }^{30}$. It has been reported that upregulation of PGC1 $\alpha$ could facilitate OXPHOS and inhibit aerobic glycolysis, which is also called the anti-Warburg effect, in several types of tumors ${ }^{13,14}$. Furthermore, PGC1 $\alpha$ has been reported to have an anti-tumorigenic function in OS. Yasuo et al. ${ }^{31}$ reported that PGC1 $\alpha$ was downregulated in OS tissue and overexpression of PGC1 $\alpha$ could promote the apoptosis of OS cells. In this study, we reveled a new mechanism that upregulation of miR-23b-3p inhibited the expression of PGC1 $\alpha$ by directly targeting it and led to a suppression of OXPHOS and a promotion of aerobic glycolysis in OS cells.

In summary, our study revealed the role and mechanism of miR-23b-3p in the OS proliferation by regulating glucose metabolism reprogram. The upregulation of miR23b-3p inhibited OXPHOS and promoted aerobic glycolysis through targeting PGC1 $\alpha$ and inhibiting its expression in OS. Consequently, targeting the miR-23b-3p/ PGC1 $\alpha$ axis may be helpful to provide new anti-cancer strategy for OS patients.

\section{Materials and methods Reagents}

Antibodies against PGC1 $\alpha$ (ab54481; Abcam, Cambridge, UK), $\beta$-actin (ab8227; Abcam, Cambridge, UK), and Ki67 (ab15580; Abcam, Cambridge, UK) were used.

\section{Cell lines and culture}

The human OS cell lines U-2OS, MNNG-HOS, Saos-2, and MG63, and human osteoblast cell line hFOB1.19 were purchased from the Cell Bank of Shanghai Institute of Biological Science (Shanghai, China). MNNG-HOS and MG63 were incubated with Eagle's minimum essential medium (Gibco, USA), whereas Saos-2 and U-2OS were incubated with McCoy's 5a Medium (Gibco, USA) containing $10 \%$ fetal bovine serum at $37^{\circ} \mathrm{C}$ in the presence of $5 \% \mathrm{CO}_{2}$. hFOB1.19 were incubated with F12 Medium (Gibco, USA) at $34.5{ }^{\circ} \mathrm{C}$ in the presence of $5 \% \mathrm{CO}_{2}$.

\section{Plasmid transfection}

Lentiviral vectors expressing PGC1 $\alpha$, miR-23b-3p shRNA, PGC1 $\alpha$ shRNA, and control construct were purchased from GenePharma (Shanghai, China). Stable cell lines overexpressing PGC1 $\alpha$, PGC1 $\alpha$ shRNA, or/and miR-23b-3p shRNA were established by infection with the lentiviruses respectively with polybrene (GM-040901B, Genomeditech, Shanghai, China) according to the manufacturer's instructions. The infected cells were selected using puromycin or blasticidin. miR-23b-3p mimics were purchased from GenePharma (Shanghai, China).

\section{RNA extraction and quantitative real-time PCR}

Trizol reagent (Sigma, USA) was used to extract total cellular RNA containing miRNA from cultured cells. SYBR $^{\circledast}$ Premix Ex Taq ${ }^{\text {Tax }}$ kit (Takara Bio, Japan) was used to quantify the transcripts of target mRNA via the Applied Biosystems 7500 Real-Time PCR system (Thermo Fisher Scientific, Inc.). Target miRNA was reverse transcribed to cDNA and expression level was determined through a miRNA qRT-PCR Sybgreen Detection Kity (A2030A003, Bio-TNT Biotechnologies, China) according to the manufacturer's instructions. The reaction was performed with one cycle of $95^{\circ} \mathrm{C}$ for $5 \mathrm{~min}$ and 40 cycles of $95^{\circ} \mathrm{C}$ for $5 \mathrm{~s}$, $60^{\circ} \mathrm{C}$ for $30 \mathrm{~s}$ using the Applied Biosystems 7500 RealTime PCR system (Thermo Fisher Scientific, Inc.). 18S and U6 were amplified as an internal reference.

\section{Western blotting}

Total cellular proteins were extracted from the target cells by RIPA lysis buffer (Beyotime, Shanghai, China) according to the manufacturer's instructions. Equal amounts of proteins were loaded onto $10 \%$ Tris-glycine SDS-polyacrylimide gel electrophoresis gels (Bio-Rad Laboratories, CA, USA). Then the separated proteins were 
A

C

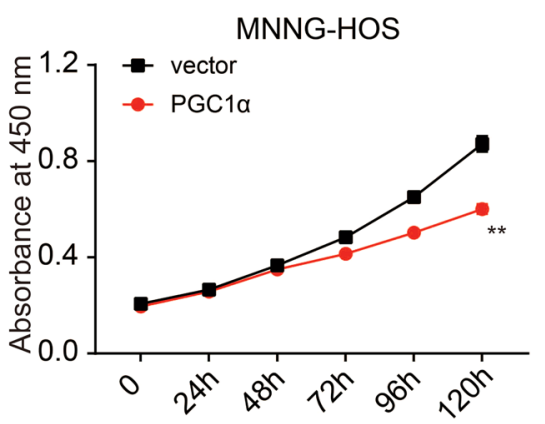

E

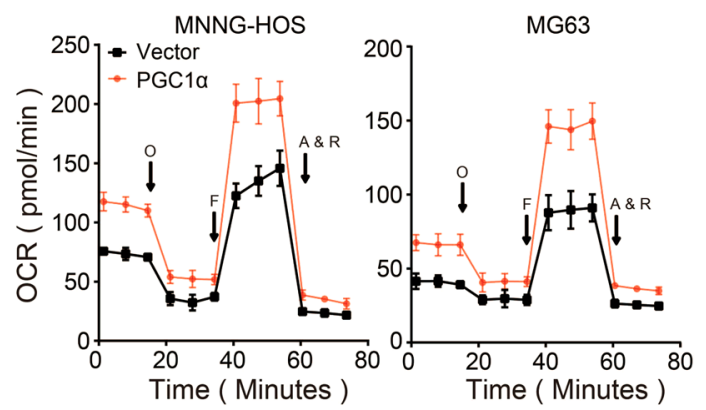

G

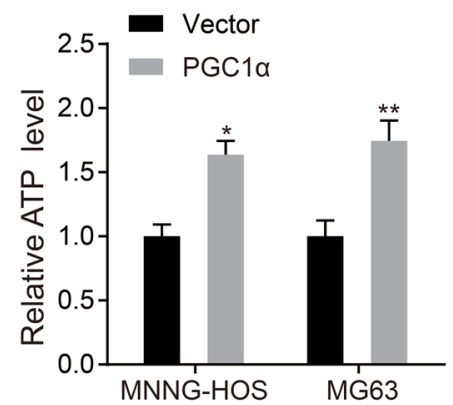

D
$\mathrm{H}$

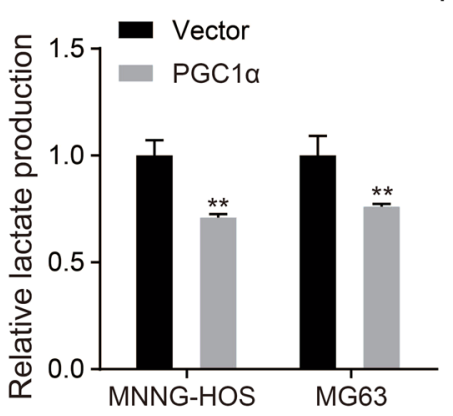

I
B

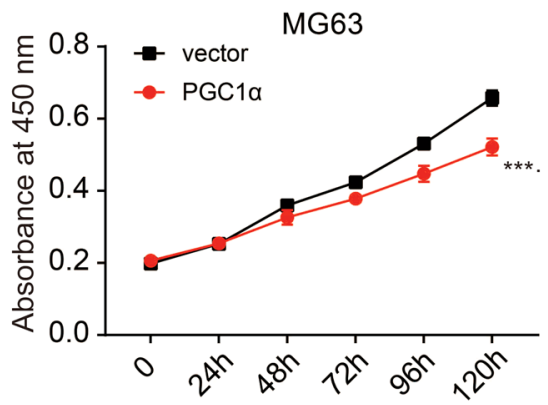

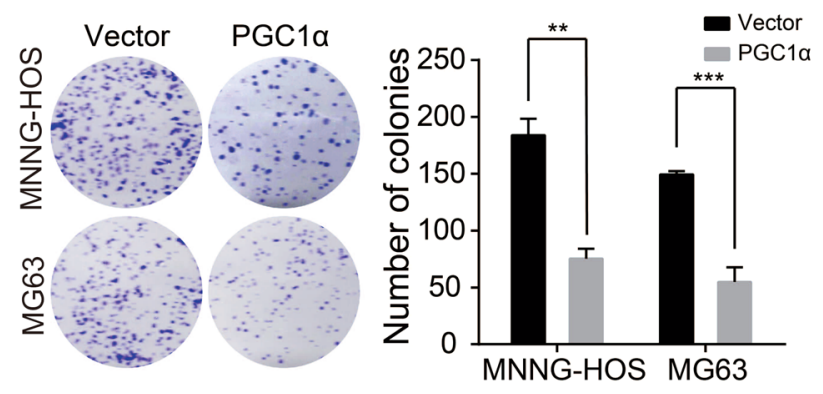

F


Fig. 3 PGC1a inhibited OS proliferation and elicited anti-Warburg effect in OS cells. a Overexpression efficacy of PGC1a in OS cells (MNNG-HOS and MG63) was detected by western blotting. b, $\mathbf{c}$ Overexpression of PGC1a inhibited MNNG-HOS and MG63 cell proliferation using the cell counting kit (CCK)-8 assay. Values are means $\pm S D,{ }^{* *} p<0.01,{ }^{* * *} p<0.001$. d Upregulation of PGC1a suppressed MNNG-HOS and MG63 cell proliferation using colony-formation assay. Values are means $\pm S D,{ }^{* *} p<0.01,{ }^{* * *} p<0.001$. e, $\mathbf{f}$ OCR and ECAR of MNNG-HOS and MG63 cells in control and ov-PGC1a group were detected. $\mathbf{g}, \mathbf{h}$ ATP level and lactate production was determined in MNNG-HOS and U-2OS cells stable overexpression PGC1a. Values are means $\pm S D,{ }^{*} p<0.05,{ }^{* *} p<0.01$. i Kaplan-Meier analysis of metastasis-free survival rate related to the expression of PGC1a expression in 88 OS cases based on a human osteosarcoma gene expression database. (https://hgserver1.amc.nl/cgi-bin/r2/main.cgi) 
A



C



E

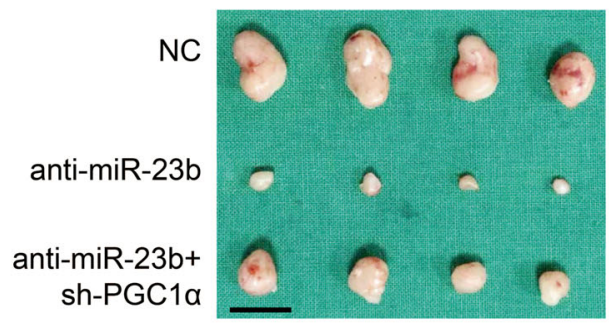

G
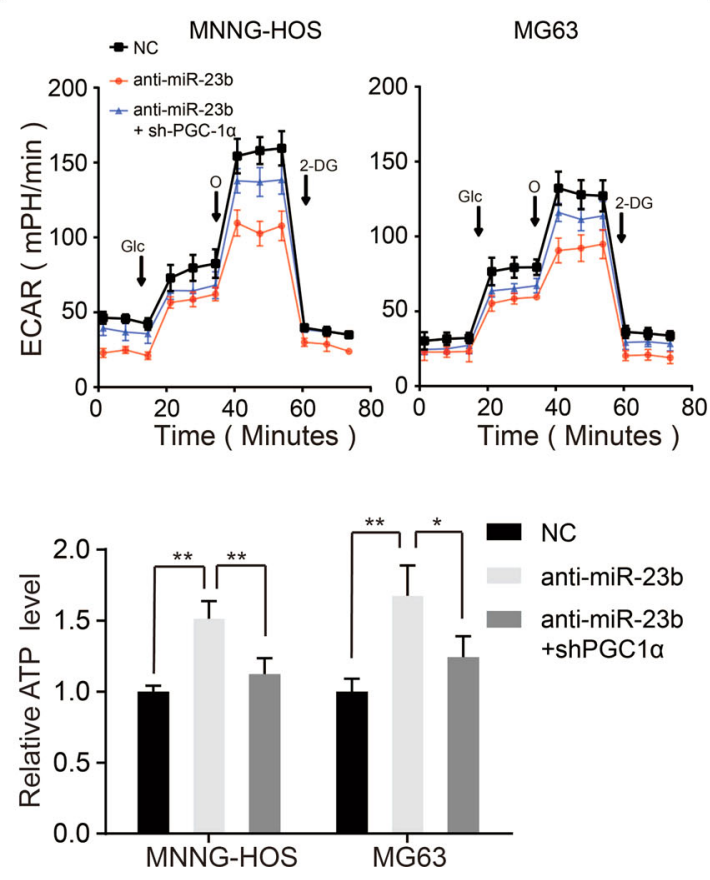

B

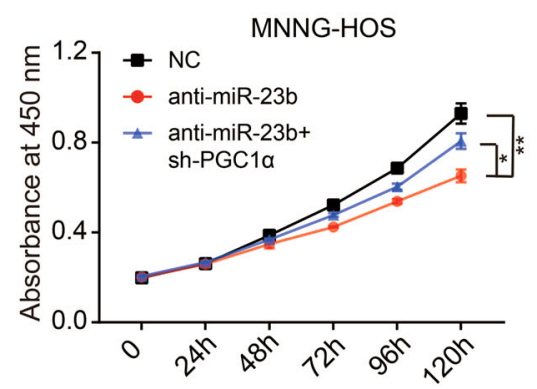

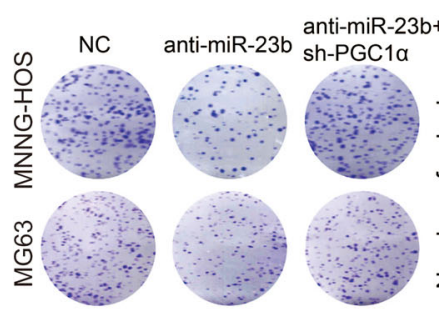

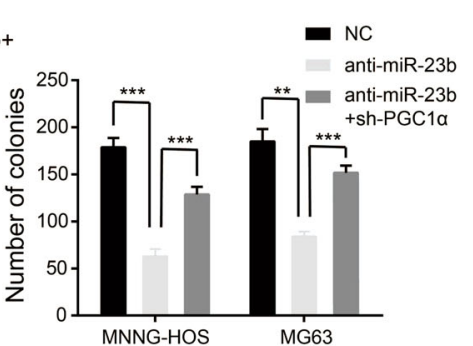

F

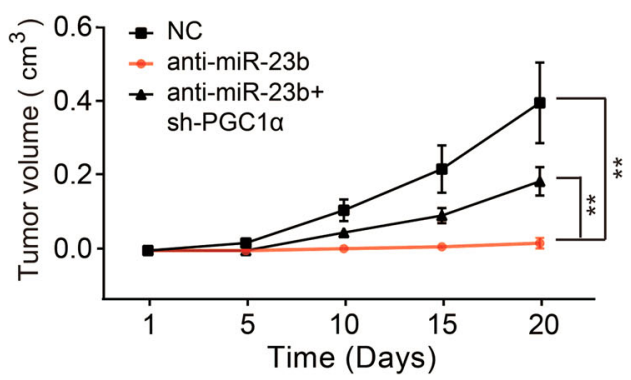

$\mathrm{H}$

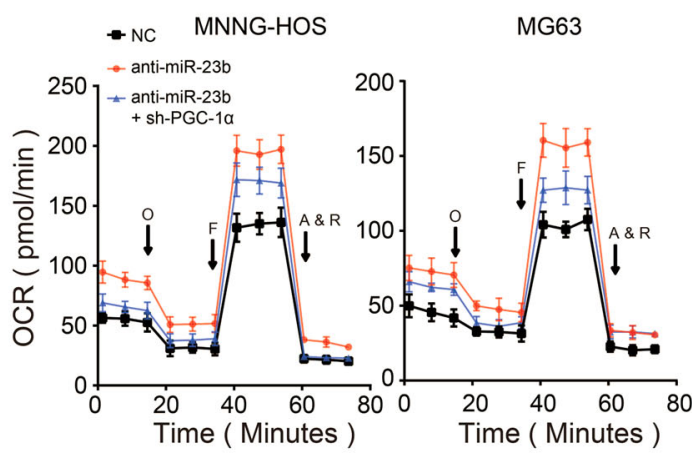

J

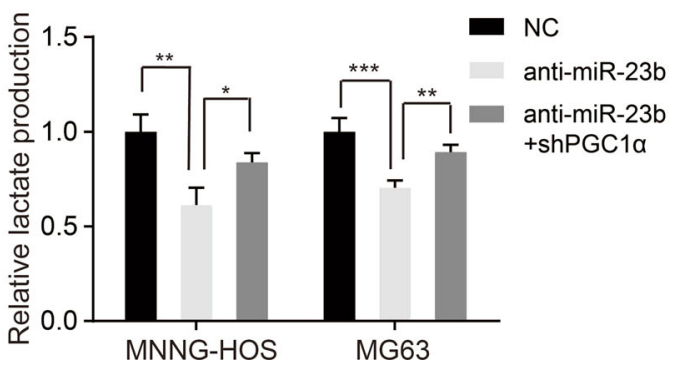

Fig. 4 (See legend on next page.) 


\begin{abstract}
(see figure on previous page)
Fig. 4 Knockdown of PGCla partly rescued the effect of knockdown of miR-23b in OS. a Knockdown efficacy of PGC1a in miR-23b-3p knockdown MNNG-HOS and MG63 cells were determined by western blotting. b, c PGC1a knockdown partly reversed the inhibitory effects of miR-23b-3p knockdown on the colony-formation properties of OS (MNNG-HOS and MG63) cells, values are means \pm SD, ${ }^{* *} p<0.01,{ }^{* * *} p<0.001$. d Silencing of PGC1a partly reversed the inhibitory effects of miR-23b-3p knockdown on the CCK-8 assay of MNNG-HOS and MG63 cells, values are means \pm SD, ${ }^{*} p<0.05,{ }^{* *} p<0.01$. e PGC1 a knockdown partly reversed the inhibitory effects of miR-23b-3p silencing on the proliferation properties of MNNG-HOS cells. Excised tumors from different groups are shown $(n=4)$. Scale bars $=1 \mathrm{~cm}$. f PGC1a knockdown partly rescued the inhibitory effects of miR-23b$3 p$ knockdown on the growth rate in MNNG-HOS cells in vivo. Tumor volumes were measured with calipers every 5 days, values are means \pm SD, ${ }^{* *} p<0.01 . \mathbf{g}$, h Altered level of OCR and ECAR in OS cells (MNNG-HOS and MG63) in different groups (Control, knockdown-miR-23b-3p, knockdownmiR-23b-3p, and knockdown- PGC1a). Values are means \pm SD. $\mathbf{i}, \mathbf{j}$. ATP level and lactate production were determined in different groups (Control, knockdown-miR-23b-3p, knockdown-miR-23b-3p, and knockdown-PGC1a). Values are means \pm SD, ${ }^{*} p<0.05,{ }^{* *} p<0.01,{ }^{* *} p<0.001$
\end{abstract}

transferred onto nitrocellulose membranes (Millipore, MA, USA). After blocking with 5\% non-fat milk, the membranes were incubated with a primary antibody at $4{ }^{\circ} \mathrm{C}$ overnight. The membranes were further incubated with secondary antibody and protein signals was detected under the ECL detection kit (Share-bio, Shanghai, China).

\section{CCK-8 assay}

Cell proliferation was measured by a CCK-8 kit (CK04, Dojindo Molecular Technologies, Kumamoto, Japan) according to the manufacturer's instructions. Cells were inoculated into 96-well plates $(3 \times 103$ cells/well $)$ and then added $10 \mu \mathrm{L}$ of CCK-8 reagent into each well at $0,24,48$, 96, and $120 \mathrm{~h}$. Absorbance at $450 \mathrm{~nm}$ was measured using a spectrophotometric plate reader. Each experiment was done in triplicate for three times.

\section{Colony-formation assays}

Target cells were seeded at a density of $1000 /$ well into 6-well plate. Two weeks later, colonies were washed with phosphate-buffered saline (PBS) twice and fixed with $4 \%$ paraformaldehyde. At the end, colonies were stained by crystal violet and counted.

\section{Cell cycle analysis}

Target cells were collected and washed with cold PBS and fixed in ice-cold $70 \%$ ethanol at $4{ }^{\circ} \mathrm{C}$ overnight. The target cells were recovered by centrifugation at 800 r.p.m. for $5 \mathrm{~min}$ and washed with PBS for $10 \mathrm{~min}$. Then, $1 \mathrm{ml}$ DNA staining solution and $10 \mu \mathrm{l}$ permeabilization solution (MultiSciences Biotech Co., HangZhou, China) were added and the cells were incubated for $30 \mathrm{~min}$ in dark. Cell cycle was determined and analyzed by flow cytometry through using the BD FACSCalibur platform.

\section{Mouse xenograft model}

$\mathrm{BALB} / \mathrm{c}$ nude mice (4-6 weeks old, male) were obtained from East China Normal University. All animal experiments and animal care were conducted following the Guide for the Care and Use of Laboratory Animals, Institute of Laboratory Animal Resources. MNNG-HOS cells $\left(1.5 \times 10^{6}\right)$ with stable miR-23b-3p knockdown or stable PGC1 $\alpha$ overexpression, or control plasmid resuspended in $200 \mu \mathrm{l}$ PBS were subcutaneously injected the nude mice. Tumor size and body weight of each nude mouse was measured every 5 days over a period of 20 day. On day 20, the mice were killed and tumor tissues were collected, fixed, and prepared for further analysis. The volume of tumor was calculated through the following equation: tumor volume $=$ length $\times$ width $^{2} / 2$.

\section{Measurement of glucose metabolism}

Glucose flux in mitochondrial and glycolysis was measured using the Seahorse XF-96 metabolic flux analyser (Seahorse Bioscience, North Billerica, MA, USA) according to the manufacturer's instructions. OCR and ECAR were measured using Seahorse XF Cell Mito Stress Test Kit and Seahorse XF Glycolysis Stress Test Kit, respectively. Briefly, $3 \times 10^{4}$ target cells were seeded into each well of a Seahorse XF-96 cell culture microplate. After the probes were calibrated, for OCR, $1 \mathrm{mM}$ oligomycin, $1 \mathrm{mM}$ p-trifluoromethoxy carbonyl cyanide phenylhydrazone and $2 \mathrm{mM}$ antimycin A plus $2 \mathrm{mM}$ rotenone (Rote/AA) were sequentially injected, and for ECAR, $10 \mathrm{mM}$ glucose, $1 \mathrm{mM}$ oligomycin, and $80 \mathrm{mM}$ 2-deoxyglucose were sequentially injected. Data were assessed and analyzed by using Seahorse XF-96 Wave software.

\section{Measurement of ATP level and lactate production}

Cellular ATP levels were determined by using a luminescence ATP detection kit (Promega, Madison, WI) according to the manufacturer's protocol. Luminescence signals was measured by using a plate reader (PerkinElmer, Waltham, MA). ATP levels were calculated from standard curves. Extracellular lactate was detected using the lactate assay kit (BioVision, CA, USA) according to the manufacturer's instructions. All values were normalized to cellular protein level.

\section{Dual luciferase reporter assay}

For luciferase activity analysis, $1 \times 10^{5}$ MNNG-HOS or $\mathrm{U}-2 \mathrm{OS}$ cells were planted into each well of 96-well plates 
the day before transfection. The cells were transiently transfected with WT PGC1 $\alpha 3^{\prime}$-UTR or mutant PGC1 $\alpha$ $3^{\prime}$-UTR were cloned into the p-MIR-reporter plasmid, combined with either miR-23b-3p mimics or NC using Lipofectamine 2000 (Invitrogen, Carlsbad, CA, USA). Forty-eight hours later, luciferase activity was measured with a Dual-Luciferase Assay System (Promega, Madison, WI, USA).

\section{Database analysis}

Prognostic analysis of PGC1 $\alpha$ expression for OS patient survival was performed by using R2 database. The data from TargetScan and miRDB were used to analyze potential target genes of miR-23b-3p.

\section{Statistical analysis}

The data were expressed as the mean \pm SD. Prism 5.0 (GraphPad, CA, USA) was used for data statistical analyses. Differences between the control and treated groups were analyzed by using two-tailed Student's $t$-test. The differences were considered statistically significant at $p<$ $0.05\left({ }^{*} p<0.05 ;{ }^{* * *} p<0.01 ;{ }^{* * * * *} p<0.001\right)$.

\section{Acknowledgements}

We are grateful to Dr. Jun Hua (State Key Laboratory of Oncogenes and Related Genes, Shanghai Cancer Institute, Ren Ji Hospital, School of Medicine, Shanghai Jiao Tong University, Shanghai, China) for assistance with our experiments.

\section{Author contributions}

R.Z. and Y.H.M. were responsible for the concept and experimental design. R.Z., X.P.L., and Y.H.M performed the experiments, data analysis, and statistical analysis.

\section{Conflict of interest}

The authors declare that they have no conflict of interest.

\section{Publisher's note}

Springer Nature remains neutral with regard to jurisdictional claims in published maps and institutional affiliations.

Received: 6 March 2019 Revised: 17 April 2019 Accepted: 24 April 2019 Published online: 16 May 2019

\section{References}

1. Liu, X., Li, C., Zhang, L., Shi, X. \& Wu, S. Personalized identification of differentially expressed modules in osteosarcoma. Medi. Sci. Monitor 23, 774-779 (2017).

2. Lee, D. H. et al. Synergistic effect of JQ1 and rapamycin for treatment of human osteosarcoma. Int. J. Cancer 136, 2055-2064 (2015).

3. Aponte-Tinao, L., Ayerza, M. A., Muscolo, D. L. \& Farfalli, G. L. Survival, recurrence, and function after epiphyseal preservation and allograft reconstruction in osteosarcoma of the knee. Clin. Orthop. Relat. Res. $\mathbf{4 7 3}$, 1789-1796 (2015)

4. Giordano, S. \& Columbano, A. MicroRNAs: new tools for diagnosis, prognosis, and therapy in hepatocellular carcinoma? Hepatology 57, 840-847 (2013).

5. Calin, G. A. \& Croce, C. M. MicroRNA signatures in human cancers. Nat. Rev. Cancer 6, 857-866 (2006).
6. Kloosterman, W. P. \& Plasterk, R. H. The diverse functions of microRNAs in animal development and disease. Dev. Cell 11, 441-450 (2006).

7. Krol, J., Loedige, I. \& Filipowicz, W. The widespread regulation of microRNA biogenesis, function and decay. Nat. Rev. Cancer 11, 597-610 (2010).

8. Chen, $\mathrm{L}$. et al. $\mathrm{VHL}$ regulates the effects of miR-23b on glioma survival and invasion via suppression of HIF-1alpha/NEGF and beta-catenin/Tcf-4 signaling. Neuro-oncology 14, 1026-1036 (2012).

9. Hu, X. et al. miR-23a/b promote tumor growth and suppress apoptosis by targeting PDCD4 in gastric cancer. Cell Death Dis. 8, e3059 (2017).

10. Jin, L. et al. Prooncogenic factors miR-23b and miR-27b are regulated by Her2/Neu, EGF, and TNF-alpha in breast cancer. Cancer Res. 73, 2884-2896 (2013).

11. Fan S., et al. Mitochondrial miRNA determines chemoresistance by reprogramming metabolism and regulating mitochondrial transcription. Cancer Res. 79. 1069-1084 (2019).

12. Girnun, G. D. The diverse role of the PPARgamma coactivator 1 family of transcriptional coactivators in cancer. Sem. Cell Dev. Biol. 23, 381-388 (2012).

13. Chen, W. et al. RIP1 maintains DNA integrity and cell proliferation by regulating PGC-1alpha-mediated mitochondrial oxidative phosphorylation and glycolysis. Cell Death Differ. 21, 1061-1070 (2014).

14. Liu, W. et al. Metastasis suppressor KISS1 seems to reverse the Warburg effect by enhancing mitochondrial biogenesis. Cancer Res. 74, 954-963 (2014).

15. Safdar, A., Abadi, A., Akhtar, M., Hettinga, B. P. \& Tarnopolsky, M. A. miRNA in the regulation of skeletal muscle adaptation to acute endurance exercise in C57BI/6J male mice. PLoS ONE 4, e5610 (2009).

16. Ell, B. et al. The microRNA-23b/27b/24 cluster promotes breast cancer lung metastasis by targeting metastasis-suppressive gene prosaposin. J. Biol. Chem. 289, 21888-21895 (2014).

17. Zaman, M. S. et al. Inhibition of PTEN gene expression by oncogenic miR-23b$3 p$ in renal cancer. PLOS ONE 7, e50203 (2012).

18. Zhuang, L. et al. MicroRNA-23b functions as an oncogene and activates AKT/ GSK3beta/beta-catenin signaling by targeting ST7L in hepatocellular carcinoma. Cell Death Dis. 8, e2804 (2017).

19. Huang, T. T. et al. The reciprocal regulation loop of Notch2 pathway and miR-23b in controlling gastric carcinogenesis. Oncotarget 6, 18012-18026 (2015).

20. Majid, S. et al. miR-23b represses proto-oncogene Src kinase and functions as methylation-silenced tumor suppressor with diagnostic and prognostic significance in prostate cancer. Cancer Res. 72, 6435-6446 (2012).

21. Majid, S. et al. MicroRNA-23b functions as a tumor suppressor by regulating Zeb1 in bladder cancer. PLOS ONE 8, e67686 (2013).

22. Liu, H. et al. miR23b3p promotes the apoptosis and inhibits the proliferation and invasion of osteosarcoma cells by targeting SIX1. Mol. Med. Rep. 18 5683-5692 (2018)

23. Perry, J. A. et al. Complementary genomic approaches highlight the PI3K mTOR pathway as a common vulnerability in osteosarcoma. Proc. Natl Acad. Sci. USA 111, E5564-E5573 (2014).

24. Wang, D. et al. Multiregion sequencing reveals the genetic heterogeneity and evolutionary history of osteosarcoma and matched pulmonary metastases. Cancer Res. 79, 7-20 (2019).

25. Choong, P. F. et al. Heterogeneity of osteosarcoma cell lines led to variable responses in reprogramming. Int. J. Med. Sci. 11, 1154-1160 (2014).

26. Collier, C. D. et al. Micrometastatic drug screening platform shows heterogeneous response to MAP chemotherapy in osteosarcoma cell lines. Clin. Orthop. Relat. Res. 476, 1400-1411 (2018).

27. Koppenol, W. H., Bounds, P. L. \& Dang, C. V. Otto Warburg's contributions to current concepts of cancer metabolism. Nat. Rev. Cancer 11, 325-337 (2011).

28. Altman, B. J., Stine, Z. E. \& Dang, C. V. From Krebs to clinic: glutamine metabolism to cancer therapy. Nat. Rev. Cancer 16, 773 (2016).

29. Giang, A. H. et al. Mitochondrial dysfunction and permeability transition in osteosarcoma cells showing the Warburg effect. J. Bio. Chem. 288 33303-33311 (2013).

30. Wu, M. et al. Multiparameter metabolic analysis reveals a close link between attenuated mitochondrial bioenergetic function and enhanced glycolysis dependency in human tumor cells. Am. J. Physiol. Cell Physiol. 292, C125-C136 (2007).

31. Onishi, Y. et al. Regulation of mitochondrial proliferation by PGC-1alpha induces cellular apoptosis in musculoskeletal malignancies. Sci. Rep. 4, 3916 (2014). 